\title{
POLYGENIC INHERITANCE OF DRUG-RESISTANCE IN THE BACTERIUM ESCHERICHIA COLI
}

\author{
L. L. CAVALLI AND G. A. MACCACARO \\ Department of Genetics, University, Cambridge
}

\section{INTRODUCTION}

Received 2.iii.52

THE inheritance of quantitative characters has been open to statistical investigation in higher organisms since Fisher's paper (1918) on the correlation between relatives. The evidence thus accumulated (Mather, 1949; Lerner, 1950) has favoured the view early announced (NilssonEhle, 1909; East, 1915) that the inheritance of such traits follows the rules of Mendelian inheritance, assuming that many genes contribute to the total effect. The importance of polygenic systems for higher organisms is to-day documented well enough not to need further emphasis.

An investigation of selection for drug resistance in bacteria led Demerec to postulate the existence of such polygenic systems whenever a high level of resistance could be attained only by a number of repeated selections, each determining a moderate increase of the resistance level. Demerec called " first-step " resistants, organisms isolated after a single exposure to the drug. Penicillin resistant first-steps isolated from sensitive strains of Staphylococcus aureus have as a rule acquired a minor degree of resistance and higher levels are attainable only stepwise. Streptomycin-resistant first-steps, on the other hand, showed a wide range of variation of the resistance level thus acquired, including full resistance. Contrasting these two types of behaviour, Demerec proposed for them the names of " penicillin " and " streptomycin-pattern" respectively.

The absence of a known sexual phase in Staphylococcus aureus and the other organisms in which penicillin resistance can be investigated prevents a test of the polygenic hypothesis. On the other hand, the discovery of recombination in Escherichia coli, strain K-12, by Lederberg and Tatum (1946) offered the basis for Mendelian analysis in at least one bacterial strain.

Streptomycin resistance in K-1 2 was thus investigated by Newcombe and Nyholm (1950a), by Demerec and by others and all resistant or dependent mutants tested proved to be allelic or due to very closely linked loci. No other cases of drug resistance showing the streptomycinpattern were described, unless one considers (somewhat loosely in respect of the original definition by Demerec) resistance to sodium azide, introduced as a chromosome marker by Lederberg, to belong to the streptomycin-pattern. Azide resistance also is apparently due to a single locus (according to data mostly unpublished by various authors). 
On the other hand, the "penicillin-pattern" seems a common mode of behaviour towards many drugs. It is clear enough that whenever selection leads to a gradual increase in resistance, if the mechanism is genic, polygenes must be at work. The first case that offered itself to observation in E. coli $\mathrm{K}-\mathrm{I} 2$ was resistance to nitrogen-mustard (Cavalli, unpublished).

Nitrogen-mustard resistance increased more or less continuously in successive transfers in broth tubes with increasing amounts of the drug; inheritance in crosses suggested more than one locus being involved but the extreme instability of the drug made a detailed study far too difficult.

Chloromycetin (chloramphenicol; henceforth abbreviated as Chl) was the next drug tested in $E$. coli $\mathrm{K}-12$ which showed a very definite "penicillin-pattern".

On first exposure to the drug on Chl-agar only a moderate increase can be attained, from 2 up to (seldom) 5 times the initially tolerated level (approx. $5 \gamma / \mathrm{ml}$.). On the other hand, selection by the common bacteriological procedure known as " training ", i.e. successive transfers in increasing concentrations of the drug, gave increases of resistance up to more than $1000 \gamma / \mathrm{ml}$., that is more than 200 times the initial level. Resistance seemed to increase very gradually, roughly twofold at every transfer, and thus the case seemed to provide excellent material for testing the hypothesis put forward by Demerec, namely that resistance of the " penicillin-pattern" is due to a polygenic system with no major genes involved.

\section{MATERIAL AND METHODS}

E. coli K-1 2 strains employed in this investigation were all derivatives of $5^{8-161}$, which is a biotinless-methionineless strain * and $\mathrm{Y}$-1o which is a threonineless, leucineless, Vitamin $\mathrm{B}_{\mathbf{1}}$-less strain.

TABLE I

Phenotypes of strains used in crosses (all from $\mathrm{E}$. coli $K-12$ )

\begin{tabular}{|c|c|c|c|c|c|c|c|c|c|c|}
\hline $\begin{array}{l}\text { Own } \\
\text { desig- } \\
\text { nation }\end{array}$ & $\begin{array}{l}\text { Lederberg's } \\
\text { designation } \\
\text { (or origin) }\end{array}$ & $\begin{array}{c}\text { Biochemical } \\
\text { deficiencies } \\
\text { (B) M T L B }\end{array}$ & & $\begin{array}{l}\text { St } \\
\text { me } \\
\text { Gal }\end{array}$ & $\begin{array}{l}\text { ar } \\
\text { atio } \\
\text { Mal }\end{array}$ & & $\begin{array}{l}\text { res } \\
V_{I}\end{array}$ & $\begin{array}{l}\text { s } \\
\text { ces } \\
\text { V6 }\end{array}$ & $\begin{array}{l}\text { res } \\
\mathrm{Az}\end{array}$ & $\begin{array}{l}\text { g } \\
\text { ices } \\
\text { St }\end{array}$ \\
\hline 2 & $58-161$ & --+++ & + & + & + & + & $\mathbf{s}$ & $\mathbf{s}$ & $\mathbf{s}$ & $\mathbf{s}$ \\
\hline 26 & W-705 & --+++ & - & - & - & + & $\mathbf{r}$ & s & s & s \\
\hline 30 & $W-583$ & $++\cdots$ & - & - & - & - & $\mathbf{r}$ & $\mathbf{s}$ & $\mathbf{s}$ & s \\
\hline 36 & $Y-10$ & $++-\cdots$ & + & + & + & + & $\mathbf{s}$ & $\mathbf{s}$ & 8 & $\mathbf{s}$ \\
\hline 47 & W-677 & $++-\cdots-$ & - & - & - & - & $\mathbf{r}$ & $\mathbf{s}$ & $\mathbf{s}$ & $\mathbf{s}$ \\
\hline 98 & from $Y-10$ & ++-- & + & + & + & + & $\mathbf{s}$ & $\mathbf{r}$ & $\mathbf{s}$ & $\mathbf{s}$ \\
\hline 100 & from $5^{8-161}$ & $-\cdots++$ & + & + & + & + & $\mathbf{s}$ & $\mathbf{r}$ & $\mathbf{r}$ & $\mathbf{s}$ \\
\hline 102 & from W-677 & $++-\cdots$ & - & - & - & - & $\mathbf{r}$ & $\mathbf{r}$ & $\mathbf{r}$ & $\mathbf{s}$ \\
\hline 123 & from Y-10 & ++--- & + & + & + & + & $\mathbf{s}$ & $\mathbf{s}$ & $\mathbf{s}$ & $\mathbf{r}$ \\
\hline
\end{tabular}

* Although 58-161 was stated to be biotinless, this deficiency was actually either lost or undetectable in the strain in our hands, since 58-161 grows well on minimal supplemented with dl-methionine alone. Absence of biotin from constituents of the medium was tested by means of Lactobacillus casei assay. However the methionine requirement is an excellent marker, even used singly, as back-mutants are exceedingly rare on control plates. 
These were the markers used for recombination, which was nearly always carried out by inoculating $10^{8}-10^{9}$ cells of each strain on the surface of minimal agar plates, prepared with the medium suggested by Lederberg, and occasionally supplemented with Vitamin $B_{1}$.

Table 1 gives the phenotypes of $5^{8-161,}$ Y-10 and some derivatives of them kindly sent by $\mathrm{J}$. Lederberg or prepared in this laboratory, which were employed throughout these experiments (markers not used were excluded).

Prototrophs, i.e. colonies growing on the minimal medium, were scored for segregating markers according to the methods given by Lederberg (1947). In most crosses however, in order to avoid parental contamination, which might have been of nuisance for resistance testing, each prototrophic colony was plated on complete agar, one colony picked at random and tested for prototrophism while scored for markers. Non-prototrophic colonies isolated in these conditions were exceptional. Care was taken to avoid unconscious selection due to colony size and shape by this procedure, as well as when picking prototrophs from the plates where crosses had taken place.

The following linear order, as after Lederberg (1947) with minor additions was found to be a satisfactory guide to interpret the results of the crosses:

$$
\mathrm{B}_{1}-\text { (B) } \mathrm{M}-\mathrm{Gal}-\mathrm{V}_{\mathbf{6}}-\mathrm{Lac} \mathrm{V}_{1}-\mathrm{Az}-(\mathrm{TL})
$$

while Xyl, Mal, Ara, were found to be linked in a complex, unexplained way with M (see for instance Newcombe and Nyholm, 1950; Cavalli, 1950).

Gal refers to the Gal locus of strain $W_{5} 83$. Such a mutation is not allelic with the Gal factors of $W_{67}$ and $W_{705}$, which are linked with Xyl, Mal, Ara.

Although the interpretation of the results indicated below is by no means dependent on the above hypothesis, this last was found to be a useful aid, in wait of an improved understanding of the situation.

Therefore the patterns of selection of recombinants were the following:

(i) when selecting full prototrophs, i.e. on minimal agar:

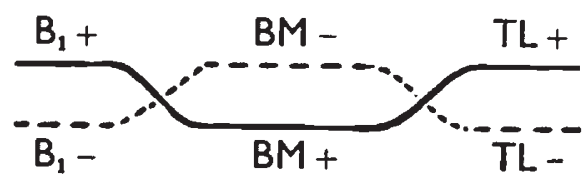

while (ii) when selecting on agar supplemented with Vitamin $B_{1}$, the majority (9o per cent) of the recombinants are obtained by exchange of the type:

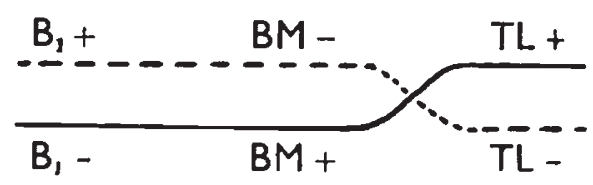

where solid lines indicate the recombinants selected for, and dotted lines indicate the hypothetical multiply deficient recombinants.

Resistance tests were carried out, except when otherwise stated, by streaking on agar plates containing various concentrations of Chloromycetin.*

At the beginning, a 2 -fold increase in concentration was used starting froms $1.25 \gamma / \mathrm{ml}$; in later experiments a scale of concentrations with a logarithmic increase of $1 / 9$ was introduced starting from $1 \gamma / \mathrm{ml}$. or higher, as it was found that the streaking

* Chloromycetin can be added and sterilised in autoclave without appreciable reduction of activity, which makes the preparation of Chloromycetin agar straightforward. Chloromycetin, Parke and Davis, was especially but not exclusively employed. 
technique could bear such a refinement, provided the cell concentration of suspensions and amounts streaked were standardised in a satisfactory way.

Parallel streaks of different resistant and sensitive organisms did not show any interaction due to Chl destruction or similar phenomena.

Moreover, although Chl can be destroyed by bacteria, as is well known (Smith and Worrell, and others), out of three highly resistant strains only one showed an increased capacity to destroy Chl, as compared with the sensitive strains (Magistretti, unpublished). Destruction can be achieved only by very high concentrations of bacteria.

Growth of streaks on $\mathrm{Chl}$ agar was scored after 24 and/or $4^{8}$ hours. A very high correlation between the observations at the two times was found. However, scoring at 48 hours was preferred, since growth in presence of the drug is delayed.

When more accurate tests of resistance were wanted, suitably diluted suspensions of the strains to be tested were plated on agar with various concentrations of Chl, and in controls without the drug and colony counts made after 3 days of incubation.

The symbolism adopted for Chl-resistant strains is $\mathrm{Chl}^{\mathrm{r}}$; when a number precedes the letter $r$ in the superscript it refers to the highest tolerated level, when it follows $r$ it denotes the number of the resistant strain (e.g. Chl ${ }^{320 r}$, i.e. a strain resistant to $320 \mathrm{\gamma} / \mathrm{ml}$., and Chlr11, i.e. resistant strain No. 11 ). The normal level of Chlsensitivity can be indicated by $\mathrm{Ch}^{\mathrm{s}}$, but this symbol is usually omitted.

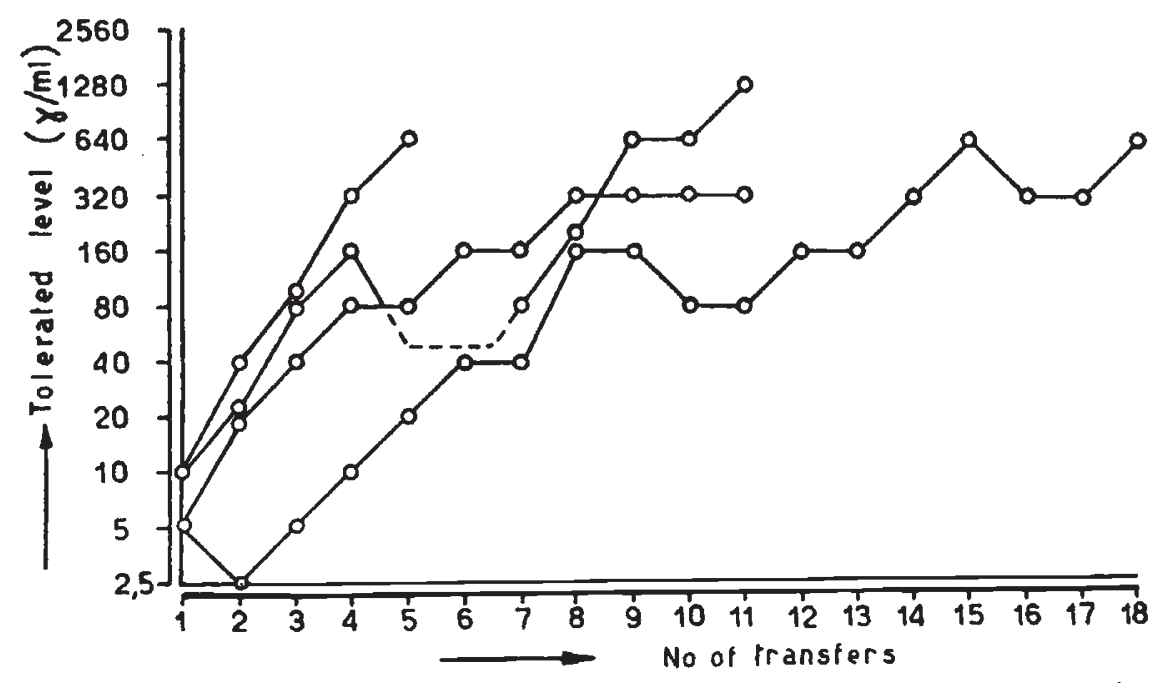

Fig. 1.-" Training" to high resistance to chloroamphenicol (Chl) by successive transfers in a series of concentrations of the drug in broth. Abscissae: number of transfers; ordinates: maximum tolerated concentration in $\gamma / \mathrm{ml}$. Four independent experiments.

\section{3. "TRAINING" TO HIGH RESISTANCE}

K-12 strains can grow in broth containing about $5 \gamma / \mathrm{ml}$. of $\mathrm{Chl}$, often with some delay. With heavy inocula growth can occur even in concentrations as high as $20 \gamma / \mathrm{ml}$. Subculturing on to higher and higher concentrations " training" to higher concentrations is regularly observed.

Fig. I shows increases in resistance such as were observed in four such independent experiments. As growth is usually delayed, especially with high concentrations, cultures were incubated 48 hours before every transfer ( $.05 \mathrm{ml}$. in ro $\mathrm{ml}$.). 
When the highest tolerance levels are reached growth becomes scanty with, as without, the drug. As can be seen from the curves in fig. I the progress towards high resistance is often smooth, although regression to lower resistance can be observed. No clear-cut steps are recognisable. The highest levels thus reached were not far from the water solubility limit of Chl, about 2 per cent according to our experience. When highly resistant strains were transferred on Chl-free media their resistance decreased from $320 \gamma / \mathrm{ml}$. to $80 \mathrm{\gamma} / \mathrm{ml}$. in one month of frequent subcultures, while if subcultures were made less frequently (once weekly or less) resistance kept to the high level, or nearly so, during the same time.

Reversion is not surprising, since highly resistant strains are impaired in their growth capacity in normal media and therefore backmutants should be favoured. The maintenance of cultures at their original resistance level for long periods seemed in fact impossible, even if cultures were kept in the refrigerator and subcultured at three months intervals. Many cultures seemed to stabilise at an intermediate level of resistance. No Chl-dependent organisms were found, although they were especially looked for by the penicillin screening technique.

Merkel Hermann and Steers, working with $E$. coli $B$, carried out selection for resistance to $\mathrm{Chl}$ in minimal media and in broth and found that in the latter case resistance was acquired more rapidly and was accompanied by several requirements for aminoacids. In strains selected by us no absolute requirements for extra growth-factors, additional to those already necessary for growth of the normal sensitive strains, were found; although growth in minimal medium, supplemented by the necessary growth-factors, was delayed in respect of controls. No appreciable alterations of other biochemical characters were found, but this experience was limited to the markers in common use for this work. The only important exception to this rule was a marked tendency of most resistant strains to give mucoid colonies on carbohydrate rich media.

Three highly resistant strains: one out of \#47 (\#47 Chl640r) and two out of \#2 (\#2 $\mathrm{Chl}^{320 \mathrm{r}}$ and \#2 Chl ${ }^{1280 \mathrm{r}}$ ) were crossed with suitable sensitive strains (\#2 and \#47 respectively). Prototrophs obtained on minimal medium were tested for Lactose fermentation, $V_{1}$ resistance and resistance to $\mathrm{Chl}$ by streaking on agar plates containing Chl from ro $\gamma / \mathrm{ml}$. to $320 \gamma / \mathrm{ml}$. or more in twofold increasing concentrations.

The distributions of the numbers of prototrophs obtained in this way are shown in table 2.

Now it is clear that if high resistance is due to a number of genes scattered along the chromosome and interacting additively, the prototrophs arising by recombination from a sensitive and a highly resistant strain should be intermediate in resistance between the parents but with a very variable degree of resistance, which should be roughly proportional for individual prototrophs to the length of the segment which the given prototroph has obtained from the resistant parent. This is 
TABLE 2

Distribution of prototrophs according to Lac $V_{1}$ phenotype and Chl-resistance in crosses: highly resistant $\times$ sensitive strains

\begin{tabular}{|c|c|c|c|c|c|c|c|c|c|}
\hline \multicolumn{10}{|c|}{ (1) Cross: \#2 2 \#47 Chl ${ }^{320 r}$} \\
\hline \multicolumn{10}{|c|}{ Chl-resistance $(\gamma / \mathrm{ml})}$. \\
\hline Lac $V_{1}$ & $<$ ro & 10 & 20 & 40 & 80 & 160 & 320 & Totals & $\begin{array}{l}\text { Geometric } \\
\text { means of } \\
\text { resistance }\end{array}$ \\
\hline$+\mathrm{s}$ & 17 & 6 & 21 & 4 & 2 & I & - & 5I & 12 \\
\hline$-s$ & i & I & 7 & 12 & 3 & 2 & - & 26 & 35 \\
\hline$-\mathbf{r}$ & - & 2 & 8 & 10 & 10 & 6 & - & $3^{6}$ & 49 \\
\hline$+\mathbf{r}$ & $\mathbf{I}$ & 2 & I & - & - & $\mathbf{I}$ & - & 5 & 18 \\
\hline Totals & 19 & II & 37 & 26 & 15 & ro & - & 118 & 25 \\
\hline \multicolumn{10}{|c|}{ 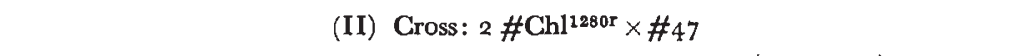 } \\
\hline Lac $V_{1}$ & $<$ I0 & 10 & 20 & 40 & 80 & 160 & 320 & Totals & \\
\hline$+s$ & 10 & 20 & 2 & 13 & 5 & - & - & 50 & 18 \\
\hline$-s$ & 49 & 74 & 3 & 2 & 2 & I & - & I3I & $8 \cdot 4$ \\
\hline$-r$ & 43 & $3^{0}$ & 3 & 2 & I & - & - & 79 & $7 \cdot 5$ \\
\hline$+\mathbf{r}$ & 2 & 5 & I & 3 & -8 & - & - & ix & 14 \\
\hline Totals & 104 & 129 & 9 & 20 & 8 & $x$ & - & $27 \pi$ & $9 \cdot 3$ \\
\hline \multicolumn{10}{|c|}{ (III) Cross: \#2 Chl ${ }^{820 \mathrm{r}} \times \# 47$} \\
\hline Lac $V_{1}$ & $<$ 10 & 10 & 20 & 40 & 80 & 160 & 320 & Totals & \\
\hline$+s$ & 8 & 13 & 13 & 12 & 3 & - & - & 49 & 17 \\
\hline$-s$ & 75 & 16 & 4 & 2 & I & - & - & $9^{8}$ & $6 \cdot 4$ \\
\hline$-\mathbf{r}$ & 31 & 16 & 3 & 一 & I & - & $\cdots$ & $5^{\mathbf{I}}$ & $7 \cdot 1$ \\
\hline$+\mathbf{r}$ & I & - & - & - & - & - & - & I & 5 \\
\hline Totals & $1 \times 5$ & 45 & 20 & 14 & 5 & - & - & $x 99$ & $8 \cdot 4$ \\
\hline
\end{tabular}

borne out by the experiments. In fact the average resistance (measured as geometric mean) for the class of recombinants $\mathrm{Lac}+\mathrm{V}_{1}^{8}$ in cross 1 of table 2 is less than that of recombinants Lac $-V_{1}^{g}$, which, having crossed over in the second segment, take a greater proportion of their chromosome from the $\mathrm{Chl}{ }^{\mathrm{r}} \mathrm{Lac}-\mathrm{V}_{1}^{\mathrm{r}}$ parent. $\mathrm{Lac}-\mathrm{V}_{1}^{\mathbf{s}}$ recombinants are in turn less resistant than Lac $-V_{1}^{r}$ (although this latter difference is not significant). In the reverse crosses, where the $\mathrm{Lac}+\mathrm{V}_{1}^{8}$ parent is the resistant

TABLE 3

Crosses between highly resistant strains

\begin{tabular}{|c|c|c|c|c|c|c|c|c|c|c|}
\hline \multicolumn{11}{|c|}{ (1) Cross: \#2 Chl ${ }^{320 r} \times \#_{47} \mathrm{Ch}^{120 \mathrm{r}}$} \\
\hline Lac & $V_{1}$ & $<$ ro & 10 & 20 & 40 & 80 & 160 & 320 & 640 & Totals \\
\hline & $\mathrm{s}$ & - & - & 20 & 18 & 6 & $\mathrm{I}$ & - & - & 45 \\
\hline - & $\mathbf{s}$ & 3 & - & 19 & 42 & 5 & 5 & I & - & 75 \\
\hline & $\mathbf{r}$ & 15 & - & 6 & 45 & 3 & 3 & - & - & 70 \\
\hline${ }^{+}$ & & $\frac{-}{16}$ & $=$ & 3 & 6 & - & - & - & - & 9 \\
\hline \multicolumn{11}{|c|}{ (II) Cross: \#2 Chl $1^{1280 r} \times \# 47 \mathrm{Ch}^{120 \mathrm{r}}$} \\
\hline Lac & $\mathrm{V}_{1}$ & $<$ io & 10 & 20 & 40 & 80 & 160 & 320 & 640 & Totals \\
\hline+ & s & II & 3 & 7 & 12 & 12 & 2 & - & - & 47 \\
\hline - & $\mathbf{s}$ & 4 & 6 & II & 18 & 26 & 10 & $\mathbf{I}$ & - & 76 \\
\hline - & $\mathbf{r}$ & 5 & 4 & 3 & 6 & 9 & I & - & - & 28 \\
\hline & & & - & 2 & I & 2 & - & - & - & 5 \\
\hline Total & & 20 & 13 & 23 & 37 & 49 & 13 & I & - & $15^{6}$ \\
\hline
\end{tabular}


one, $L a c-V_{1}^{8}$ are more resistant than $L a c+V_{1}^{s}$. It should be noted that the average resistance of all recombinants is always significantly smaller than the (geometric) mean resistance of the parents. The geometric scale was chosen for all comparisons because of the nearly geometric increase of resistance during selection, especially in its early phases. We shall see later that this diminution of resistance in the prototrophs is well compatible with a polygenic system, in which nonadditive interactions occur.

When highly resistant strains were crossed between themselves the distributions of prototrophs given in table 3 were found. These results are again contradictory of a simple polygenic hypothesis in which all polygenes react additively together. In fact they show that fully sensitive prototrophs can appear. This cannot be due simply to the condition that the genes in the resistant strains crossed together are all non-allelic. This would not explain why no prototroph with resistance higher than that of either parent was recorded. Also it would not explain why such fully sensitive prototrophs are, as they often are, bad growers. Rather, it is likely that either (a) negative interaction can occur between two genes for resistance, perhaps affecting growth rate as well; $(b)$ that some genes may act as "modifiers" of the resistance due to other genes, determining an increase of it, without having any effect on resistance in the absence of the genes the expressivity of which they modify.

Such hypotheses were tested by crossing three sensitive prototrophs (arising from the cross between the two highly resistant strains mentioned above) to \# $\mathrm{I}_{23}$, which is a $\mathrm{TLB}_{1}$ requiring, streptomycin resistant, Chl sensitive strain, on minimal medium supplemented with streptomycin. The Lac $V_{1}$ phenotype of the three sensitive prototrophs was $-s,-r,-r$ respectively and all of them did not grow on $10 \gamma / \mathrm{ml}$. of Chl. There is no cross resistance between $\mathrm{Chl}$ and streptomycin. The two latter prototrophs (both $-\mathrm{r}$ ) crossed to $\mathrm{TLB}_{1}-\mathrm{St}^{\mathrm{r}}$ on minimal agar supplemented with streptomycin gave all $\mathrm{Chl}$ sensitive prototrophs. The -s prototroph crossed in similar conditions to the $\mathrm{TLB}_{1}-\mathrm{St}^{\mathrm{r}}$ strain gave (out of 48 recombinants):

\section{Chl sensitives}

43 resistants to $\mathrm{I} 6 \gamma / \mathrm{ml}$ of $\mathrm{Chl}$

2 resistants to $25 \gamma / \mathrm{ml}$ of $\mathrm{Chl}$

However, when this cross was later repeated, using strains derived from 3 colonies isolated from the $-\mathrm{s}$ prototroph, one of which was a good grower and the other two poor growers, no recombinants with a resistance clearly higher than that of either parent was recorded. The problem was not analysed further on these data. The whole question of interaction between genes will be discussed later.

\section{ANALYSIS OF SINGLE STEPS}

The process of "training", i.e. in the present case the attainment of resistance by a number of successive transfers in liquid media con- 
taining higher and higher concentrations of the drug, gave rise, for Chl, to results which are best interpreted on the basis of a polygenic system. If it is possible to obtain resistant individuals, in which one single genedifference is responsible for resistance, they should give, on crossing to sensitives, clear-cut segregations into resistants-all showing the same level of resistance-and full sensitives, in marked contrast with the results obtained with strains derived by means of long selection, i.e. " training".

Such a single-step-resistant should be obtainable either by (i) stopping the training process after the first exposure to the drug, or (ii) by plate selection procedures such as were used by Demerec in his first investigations, and by others.

By the latter method a large number of cells (of the order of $\mathrm{IO}^{9}$ cells) of the sensitive strain, from cultures originated from a small inoculum, were plated on Chl-agar. Provided the concentration of the drug is between 20 and $40 \gamma / \mathrm{ml}$. a few colonies will develop after the first day of incubation and many more on the second and third day. As shown elsewhere (Cavalli, I952) colonies developing later are on average less resistant than those developed earlier. Fluctuation tests showed a high variability of the number of resistant colonies derived from parallel independent sensitive cultures; but a closer analysis according to Luria and Delbrück's model, developed by Lea and Coulson, is prevented by the difficulty of obtaining reliable counts of resistant colonies from the same culture. Such difficulties are in part due to the extreme variability of size of the resistant colonies, many of which are near the threshold of visibility.

The great variability of size should be a consequence of the great variability of the time of onset of growth of individual resistants, and this is, partly at least, caused by variability of the resistance level of independent single steps.

Resistants which were presumably single steps were isolated by both techniques and crossed to sensitives.

Table 4 shows the results of such crosses for two resistants isolated after one transfer of $\# 26$ in Chl-broth $(20 \gamma / \mathrm{ml}$.) under shaking for 48 hours, showing a different degree of resistance. If a line is drawn dividing individuals growing on $5 \gamma / \mathrm{ml}$. but not on Io $\gamma / \mathrm{ml}$. and those growing on Io $\gamma / \mathrm{ml}$. and more, considering the former as sensitives, the latter as resistants, one can map a locus for Chl-resistance between $\mathrm{M}$ and $\mathrm{V}_{6}$. One thing worth noting is that the first resistant Chl ${ }^{20 r}$ which reaches the resistance level of only $20 \gamma / \mathrm{ml}$, gives a segregation which is superimposable on that obtained with the second resistant $\mathrm{Chl}^{40 \mathrm{r}}$ which did, instead, grow on $40 \gamma / \mathrm{ml}$. In either cross the resistant prototrophs show resistance to ro or $20 \gamma / \mathrm{ml}$., never higher, which is approximately the same as that shown by the first resistant parent $\mathrm{Chl}^{20 \mathrm{r}}$ (especially considering that the two normal strains \#26 and \#98 are not identical in resistance to Chl, the former being spontaneously somewhat more resistant; see later). Since the two resistants 
are not independent in origin, because they were isolated out of the same selection tube, i.e. Chl-broth, the easiest explanation is that in the condition employed for selection in liquid medium, even after one exposure a second step of resistance can be reached. Growth under shaking, as here, gives in fact excellent conditions for rapid selection and it is not impossible that in one cycle of growth in liquid medium

TABLE 4

Crosses between sensitive strains and "first step" resistants, obtained by selecting for resistance in liquid media

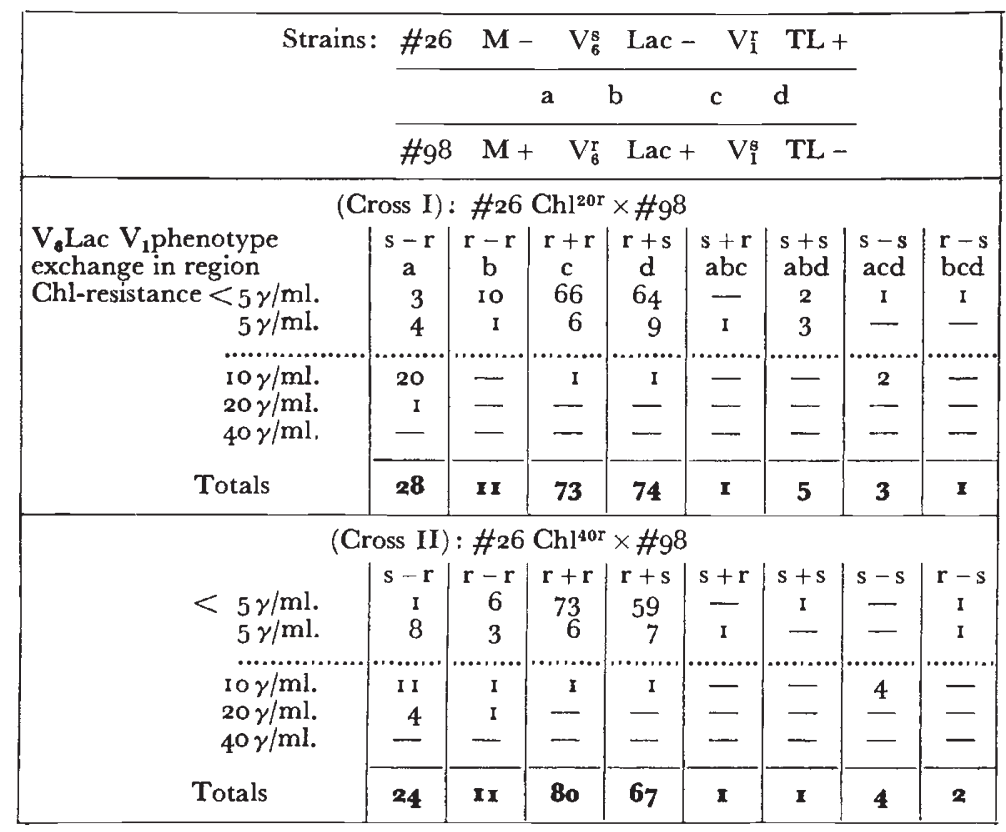

more than one step of resistance can be climbed. According to this interpretation the first resistant strain, $\mathrm{Chl}^{20 r}$, was the first step and the second resistant $\mathrm{Chl}^{40 \mathrm{r}}$ had gone one step further, the second step gene being closely linked with $\mathbf{M}$ and therefore not showing among segregants.

When first steps obtained by plate selection were analysed with crossings, results interpetable in terms of one only gene-difference were the rule, except in a case which will be discussed later.

Out of ten $\mathrm{Chl}^{\mathrm{r}}$ genes that were mapped in crosses of first or second steps with sensitives, two independent ones were closely linked with $\mathbf{M}$; one was linked with $\mathrm{M}$ but more loosely, showing some linkage with an " unmappable" marker, Gal; three were between $\mathrm{M}$ and $\mathrm{V}_{6}$; one between $V_{6}$ and Lac and one probably right of Lac; one was between $\mathrm{V}_{1}$ and TL and one (to be analysed later) was unlinked with genes between $M$ and TL.

Thus there is not a fixed locus, mutation at which is responsible for 
first step resistance, but rather a number of loci each of which can determine this effect.

The above data in which resistance was tested by the streaking technique can give satisfactory results as far as mapping is concerned, but will not answer the question whether resistance of single steps is actually segregating in a discrete way, as is to be expected for a mechanism of particulate inheritance, in contrast with the expectation of blending inheritance. To this aim more refined tests for resistance

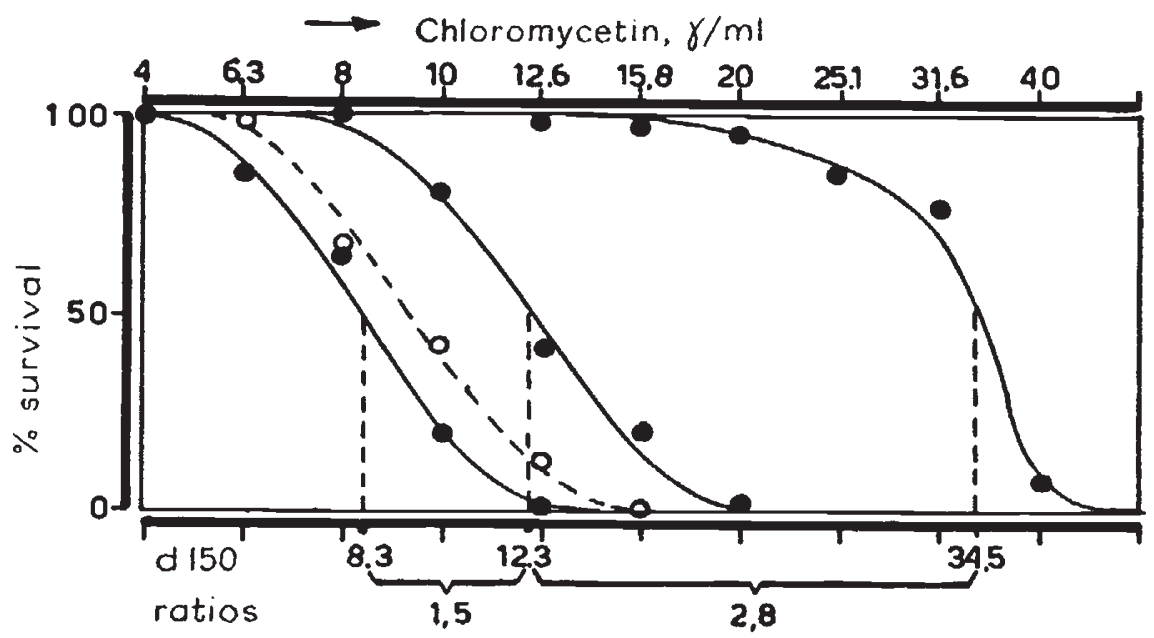

Fig. 2.-Resistance curves of four strains. Abscissae, concentration of Chl; ordinates, percentage of survival. The four curves, from left to right, belong to: \# Ioo, sensitive; \#47, sensitive (dotted line); \# roo Chlr8, first-step resistant from \# roo; \# roo Chl ${ }^{\mathrm{r} / \mathrm{rr}}$, second-step resistant from saine.

are necessary. The following was used in the present case. Resistant and sensitive strains and prototrophs from them were tested by plating a suitable number of cells (of the order I00-300) in agar plates, and in agar containing various concentrations of Chl. After three days of incubation the number of colonies developed was counted and survival "percentages" calculated as the ratios of the number of colonies on agar with a given amount of $\mathrm{Chl} / \mathrm{ml}$. and the number of colonies on drug-free agar plates.*

Survival curves obtained for two parental sensitive strains (\# Ioo and \#47) are given in fig. 2 and it can be noted that these strains show some difference of sensitivity. This slight increase of resistance seems to be often but not always associated with the Lac - $V_{1}^{r}$ phenotype and was the cause of some trouble in interpretation of segregations (sce also before, $r e$ experiments reported in table 4 ).

In fig. 2 are also shown the survival curves of the first step resistant

* These experiments were carried out in another laboratory (Istituto Sieroterapico Milanesi, Milan) and with other media, so that it is not surprising that the resistance levels have shifted from those previously reported. 
$\mathrm{Ch}^{\mathrm{r} 8}$ derived from \# Ioo by plate selection and of the second step resistant $\mathrm{Chl}^{\mathrm{r} / \mathrm{r} 9}$ derived from $\mathrm{Ch}^{\mathrm{r}}{ }^{\mathrm{8}}$ by a second plate selection. The increase in resistance observed in $\mathrm{Chl}^{\mathrm{r}}{ }^{8}$ is exceedingly slight, the $5^{\circ}$ per cent tolerated dose having increased only $I \cdot 5$ times. In spite of this a segregation of resistance is recognisable among recombinants from \# $100 \mathrm{Ch}^{\mathrm{r} 8} \times \# 47$ in the form of a bimodal distribution, as can be
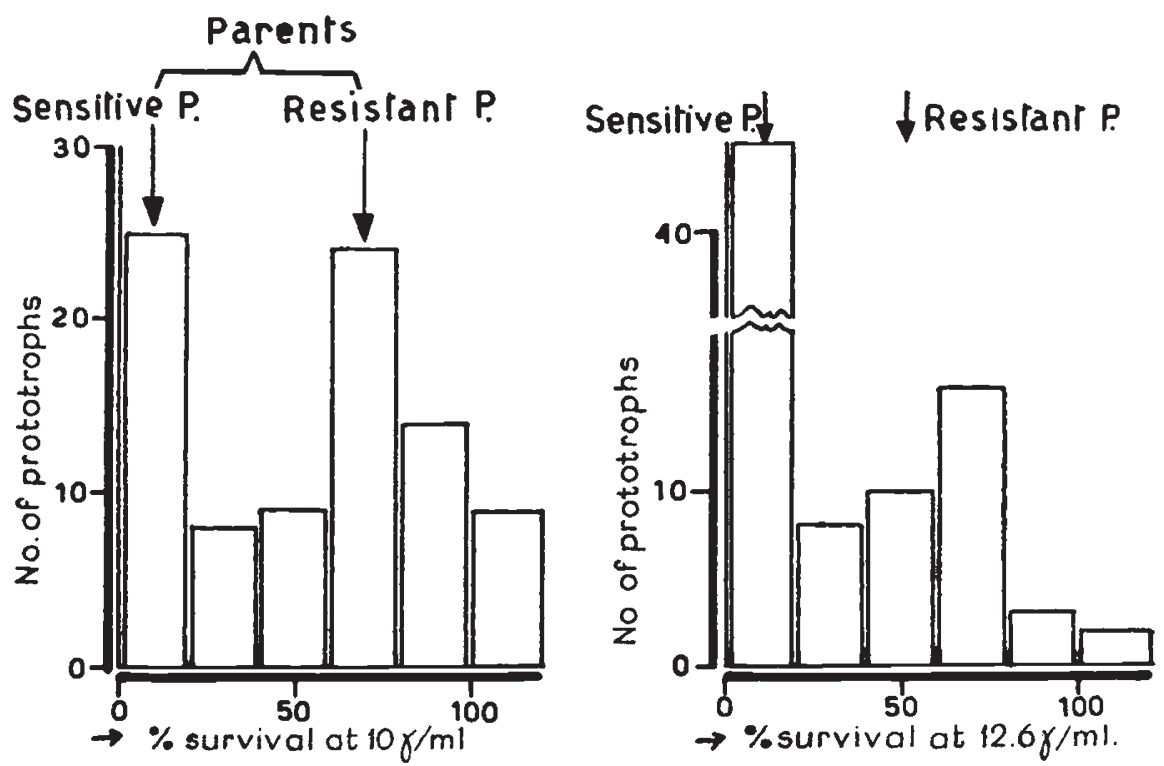

Fig. 3.-Distribution of resistance of prototrophs from cross \# $100 \mathrm{Chl} \times{ }^{18} \times 47$. Resistance is indicated on the abscissae as " percentage " of survival at a given drug concentration. In the left hand figure, resistance at 10 $\mathrm{\gamma} / \mathrm{ml}$.; in the right hand figure at $12.6 \mathrm{\gamma} / \mathrm{ml}$.

seen from figs. $3^{a}$ and $3^{b}$, representing the distribution of resistance of the prototrophs from cross \#100 $\mathrm{Chl}^{\mathrm{r} 8} \times \# 47$, where the scale in which resistance is measured is survival percentage at a given concentration of the drug. Two drug levels are indicated (10 and $12.6 \mathrm{r} / \mathrm{ml}$.). In the histograms the arrows indicate the resistance levels of the two parents and it can be seen that they correspond approximately with the two modes. It is not surprising that the distribution of resistance of the prototrophs, although being bimodal, is not clearly discontinuous. The difference in resistance between the parents is small, in comparison with the standard deviation due to error in the measure of the individual resistance (about \pm Io per cent). Also the existence of some difference in resistance between \#IOO (the normal parent of Chl ${ }^{\mathbf{8}}$ ) and \#47 will undoubtedly contribute to obscure the results.

If an arbitrary distinction is made at a survival of say 20 per cent, in fig. $3 a$, to classify sensitives and resistants, no linkage between resistance and the usual Lac $V_{1}$ markers is found (data not shown). This means, as far as present linkage tests can say, that the gene for resistance in strain $\mathrm{Chl}^{\mathrm{r}}{ }^{8}$ is outside the marked region. When the second step 
$\mathrm{Chl}^{\mathrm{r} / \mathrm{r} 9}$ is crossed to sensitive $\# 47$ and the distribution of resistance of prototrophs is analysed in the same way (fig. 4) a more complex distribution is expected and indeed found.
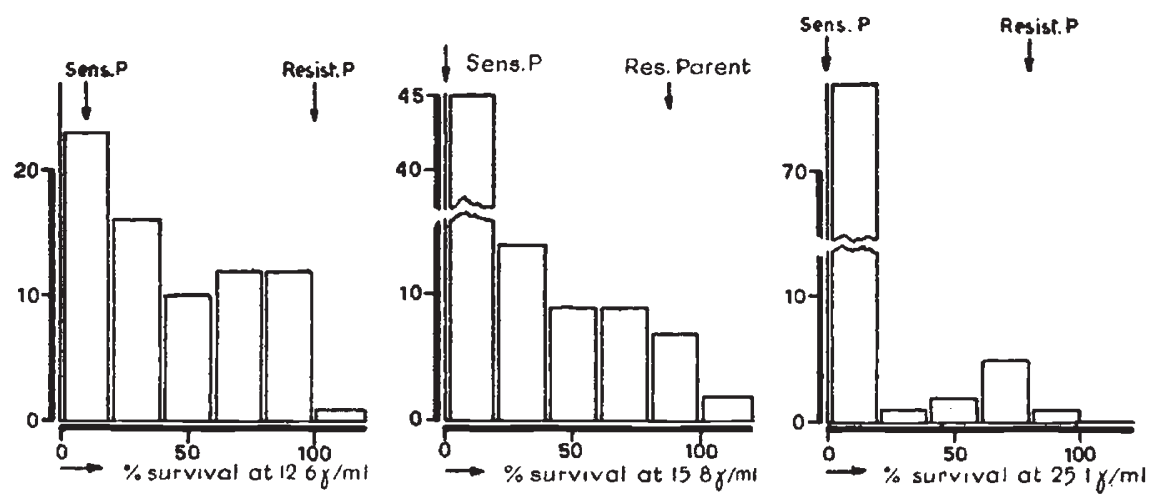

F1c. 4.-Distribution of resistance of prototrophs from cross \#100 Ch${ }^{\mathrm{r} 8 / \mathrm{r} 8} \times \# 47$. Abscissae and ordinates as in fig. 3 .

Figs. $4 a, b, c$ show such distributions as they result from the analysis at three different levels of drug concentration. Four phenotypes $\left(\mathrm{Ch}^{\mathrm{s}}\right.$, $\mathrm{Chl}^{\mathrm{r} 8}, \mathrm{Chl}^{\mathrm{r} 8}, \mathrm{Chl}^{\mathrm{r8} / \mathrm{rg}}$ ) are expected in this cross, but it would be difficult to recognise them all. At the highest concentration tested, however, the action of the second step gene is clearly recognisable, giving a mode at the resistance level just below that expected according to the resistance level of the $\mathrm{Chl}^{\mathrm{r} / \mathrm{rg} 9}$ parent (as shown by the arrow). We can assume-somewhat arbitrarily of course- - that 20 per cent survival at $25 \mathrm{r} / \mathrm{ml}$. represents the resistance level which divides recombinants of this cross into those possessing the second step gene $\mathrm{Ch}^{\mathrm{r}}{ }^{\mathrm{9}}$ (irrespectively

TABLE 5

Cross with \# roo $\mathrm{Ch}^{\mathrm{s}}$ of second step resistant \# roo Chl $\mathrm{r}^{\mathrm{r} / \mathrm{r}} \mathrm{s}$

Chl-resistance scored according to survival at $25 \gamma / \mathrm{ml}$; Chl ${ }^{r}$, prototrophs with survival percentage $>20$ per cent, $\mathrm{Ch}^{8}$ at survival percentage less than 20 per cent.

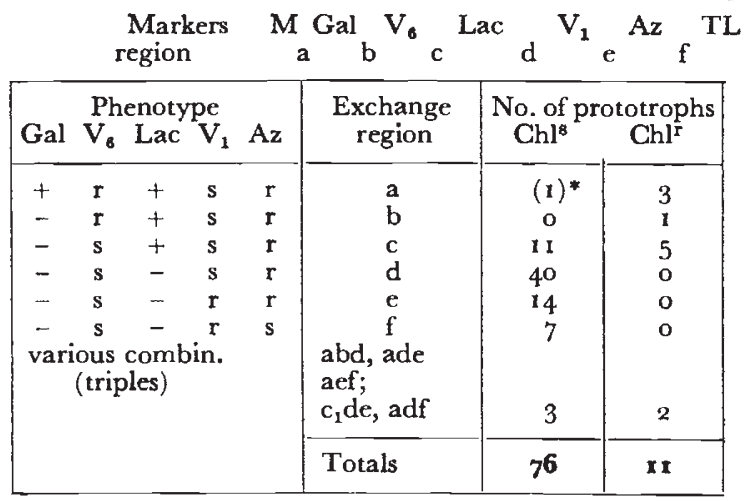

* uncertain classification for resistance 
of whether they also possess the first step $\mathrm{Ch}^{\mathrm{r}}{ }^{\mathrm{r}}$ ) and those not possessing it. If we score prototrophs $\mathrm{Ch}^{\mathrm{rg}}$ and $\mathrm{Chl}$ according to this assumption, and examine their distribution according to $\mathrm{Ch}^{\mathrm{B}}$ and the other markers analysed in this cross $\left(\mathrm{Gal} \mathrm{V}_{6} \mathrm{Lac} \mathrm{V}_{1}\right)$ we find that $\mathrm{Chl}^{\mathrm{r} 8}$ must be between $V_{6}$ and Lac (see table 5). The data are scanty because of the tediousness of the procedure of determining resistance.

\section{ALLELISM AND INTERACTION}

From the previously reported experiments a relatively large number of genes seems responsible for behaviour in respect of Chl-resistance in E. coli K-12. Only a few preliminary tests were made in order to ascertain (i) how often are two independent mutants allelic, (ii) what interaction can one expect between non-allelic genes.

The following experiment is a contribution to the problem (i): Ten independent first steps were selected from $\# 2$ by plating fully grown cultures started from about roo cells each on Chl-agar containing $25 \mathrm{\gamma} / \mathrm{ml}$. Similarly seven such first steps were selected from \#47: one first step from \#47 was crossed with each of ten first steps from \#2 and the proportion of sensitive prototrophs thus obtained was determined.

The results are given in table 6 .

TABLE 6

Allelism tests of first steps

Percentages of Chl-sensitives and number of prototrophs tested in 15 crosses

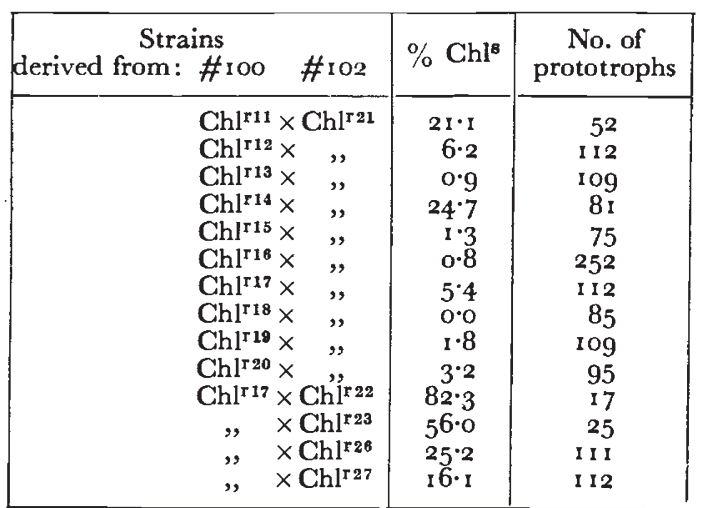

Only in one out of the 15 crosses were all prototrophs resistant and perhaps even in this case-had more recombinants been investigateda few sensitives might have arisen. Moreover there is a significant difference between the proportions of sensitives obtained in the various crosses.

At least four or five different loci should be invoked in order to explain the results: one locus for $\mathrm{Ch}^{\mathrm{r21}}$ and $\mathrm{Ch} \mathrm{l}^{\mathrm{r1}}$, one locus for $\mathrm{Ch} \mathbf{l}^{\mathrm{r12}}$,

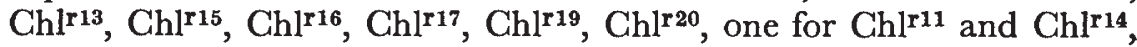
one for $\mathrm{Ch}^{\mathrm{r22}}$ and $\mathrm{Ch}^{\mathrm{r23}}$, one, perhaps, for $\mathrm{Ch}^{\mathrm{r26}}$ and $\mathrm{Chl}^{\mathrm{r27}}$, which might however be the same as Chl ${ }^{\text {r11 }}$ and Chl ${ }^{\text {r14 }}$. 
However since strains $\mathrm{Ch} \mathbf{l}^{\mathrm{ri1}-20}$ cannot be crossed together unless special markers are added by mutation, the extensive work necessary to test these conclusions on the frequency of non-allelic genes has not been carried out.

Similarly the problem of interaction between non-allelic genes was examined only to the extent that we limited our attention to the possibility of obtaining the addition, by recombination, of the resistances of independent first steps, this being a fundamental requirement of a polygenic theory.

In these experiments $\mathrm{Ch}^{\mathrm{r} 18}$ was crossed to $\mathrm{Ch}^{\mathrm{r} 22}$, $\mathrm{Chl}^{\mathrm{r} 23}$, $\mathrm{Ch}^{\mathrm{r}{ }^{25}}$ and $\mathrm{Chl}^{\mathrm{r27}}$. These strains were selected among those which kept their resistance unimpaired for a year and were reisolated before use. Crosses were made on minimal medium and prototrophs transferred by means of the velvet technique (Lederberg, personal communication) to minimal plates containing $\mathrm{Chl}$ in various concentrations. Results were as given in table 7 .

TABLE 7

Number of colonies grown on minimal agar with given amounts of Chl

\begin{tabular}{|c|c|c|c|c|c|c|}
\hline & $0 \gamma$ & $40 \gamma$ & $63 \gamma$ & $79 \gamma$ & $100 \gamma$ & \\
\hline $\left.\mathrm{Ch}^{\mathrm{r} 18}=\mathrm{Ch}\right]^{\mathrm{r} 22}$ & 132 & 50 & 22 & & 8 & \\
\hline $\mathrm{Ch}^{118}=\mathrm{Ch}^{1223}$ & 55 & 37 & 22 & $\begin{array}{r}5 \\
2\end{array}$ & 0 & $\times 6$ \\
\hline $\mathrm{Ch}^{118}=\mathrm{Ch}^{\mathrm{r} 25}$ & 443 & 164 & 13 & 10 & 8 & $63^{8}$ \\
\hline $\mathrm{Ch}^{1 \mathrm{r}^{18}}=\mathrm{Ch}^{\mathrm{r} \mathrm{r}^{27}}$ & $\begin{array}{l}159 \\
159\end{array}$ & 73 & 13 & 12 & 5 & 262 \\
\hline & 789 & 324 & 70 & 39 & $2 I$ & 1243 \\
\hline
\end{tabular}

The parent strains tested for resistance on complete medium showed a similar degree of resistance, both growing at $63 \gamma / \mathrm{ml}$. and not at $79 \mathrm{r} / \mathrm{ml}$. Therefore the experiments reported in the table seem to show that recombinants from independent first steps can reach a higher degree of resistance than either parent. This was checked by isolating from drug-free minimal agar the prototrophs showing apparently higher resistance, and they were found to give a resistance occasionally as high as $159 \mathrm{\gamma} / \mathrm{ml}$. Since the technique of transferring whole colonies did not prove sufficiently reliable from a quantitative point of view, one of these crosses $\left(\mathrm{Ch}^{118} \times \mathrm{Chl}^{\mathrm{r2}}\right)$ was repeated with the ordinary technique, i.e. by streaking suspensions prepared from each prototroph on plates of agar containing various concentrations of the drug (in parallel with controls, that is streaks from the sensitive parents). In two experiments, there were 3 per cent and 4 per cent of the prototrophs showing a resistance higher than that of either parent.

To control more accurately this positive interaction between independent first steps, both of them were crossed between themselves and with the (complementary) sensitive parents, i.e. the cross $\mathrm{Chl}^{\mathrm{r} 18} \times \mathrm{Ch}^{\mathrm{r} 22}$ was made in parallel with

(I) cross $\mathrm{Ch}^{\mathrm{r} 18} \times$ the normal sensitive parent of $\mathrm{Ch}^{\mathrm{r} 22}$ which is \# 102.

(2) cross $\mathrm{Ch}^{\mathrm{r22}} \times$ the normal sensitive parent of $\mathrm{Chl}^{\mathrm{r} 18}$ which is \#- roo. 
The crosses were carried out on minimal medium in absence of Chl and the prototrophs later scored for resistance and Lac $V_{1}$ phenotype. In table 8 are shown the results obtained, i.e. the distribution of resistance of the recombinants from these three crosses and their distribution according to Lac $\mathrm{V}_{1}$ phenotype.

TABLE 8

Positive interaction between independent first steps

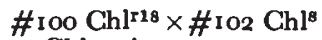

Chl resistance:

\begin{tabular}{cc|rrrrrr|r} 
Lac & $\mathrm{V}_{1}$ & $<20$ & 20 & 32 & 50 & 79 & 126 & \\
\hline+ & $\mathrm{s}$ & 19 & 25 & 3 & 7 & 3 & 0 & $\mathbf{5 7}$ \\
- & $\mathrm{s}$ & 75 & 3 & 3 & $\mathrm{I}$ & 0 & 0 & $\mathbf{8 2}$ \\
- & $\mathrm{r}$ & 45 & $\mathrm{I}$ & 2 & $\mathrm{I}$ & 0 & 0 & $\mathbf{4 9}$ \\
+ & $\mathrm{r}$ & 3 & 0 & 0 & 1 & 0 & 0 & 4 \\
\hline & & $\mathbf{1 4 2}$ & 29 & 8 & 10 & 3 & 0 & 192
\end{tabular}

\begin{tabular}{|c|c|c|c|c|c|c|c|c|}
\hline \multirow[b]{2}{*}{ Lac } & \multicolumn{8}{|c|}{ \#100 Chl ${ }^{8} \times$ \# $\mathrm{O}_{2} \mathrm{Ch}^{\mathrm{r} 22}$} \\
\hline & $V_{1}$ & $<20$ & 20 & 32 & 50 & 79 & 126 & \\
\hline+ & $\mathbf{s}$ & Io & 4 & 22 & Io & 0 & 0 & $4^{6}$ \\
\hline- & $\mathbf{s}$ & 5 & 2 & 50 & $2 I$ & 0 & 0 & 78 \\
\hline- & $\mathbf{r}$ & 3 & 3 & 29 & I 5 & 0 & 0 & $5^{\circ}$ \\
\hline+ & $\mathbf{r}$ & I & I & 3 & 3 & 0 & o & 8 \\
\hline & & rg & I0 & 104 & 49 & $\mathbf{0}$ & $\mathbf{0}$ & 182 \\
\hline
\end{tabular}

\begin{tabular}{|c|c|c|c|c|c|c|c|c|}
\hline \multirow[b]{2}{*}{ Lac } & \multicolumn{8}{|c|}{ 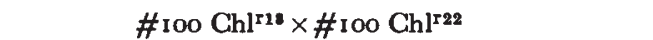 } \\
\hline & $V_{2}$ & $<20$ & 20 & 32 & $5^{\circ}$ & 79 & 126 & \\
\hline+ & $\mathbf{s}$ & I & o & 7 & 26 & 9 & 3 & $4^{6}$ \\
\hline - & $\mathbf{s}$ & 7 & 4 & 12 & 59 & 9 & & 94 \\
\hline - & $\mathbf{r}$ & 4 & 3 & 9 & 38 & o & 2 & 55 \\
\hline+ & $\mathbf{r}$ & I & o & o & 4 & 2 & 3 & 10 \\
\hline & & 13 & 7 & 28 & 127 & 20 & 11 & 205 \\
\hline
\end{tabular}

The cross $\mathrm{Ch}^{\mathrm{r} 18} \times \#$ IO2 gave a small percentage of recombinants (24 out of I92, i.e. I I per cent) which were of resistance as high as that of the Chl ${ }^{\mathrm{r} 18}$ parent (i.e. between 32 and $79 \gamma / \mathrm{ml}$.) and a majority of sensitives or slightly resistant recombinants. As it has been said before these experiments were conducted in another laboratory and with other media; therefore the absolute levels of resistance are not exactly correspondent with those seen earlier, although the relative levels are more closely correspondent.

From the distribution of resistants according to Lac $\mathrm{V}_{1}$ markers, the gene for resistance should be located closely enough to $\mathrm{BM}$ and right of it.

The cross Chl ${ }^{\mathrm{r} 22} \times \#$ I oo shows a majority of resistant recombinants, there being 153 out of 182 , i.e. 84 per cent, prototrophs with a resistance level higher than $3 \mathrm{I}$ and lower than 79, with very little if any correlation with the $\operatorname{Lac} V_{1}$ markers. Mapping of the gene involved is uneasy; however it is linked with BM, but not very closely, and perhaps left of it.

From the evidence of the crosses of the first step resistant with sensitive strains it would be predicted that II per cent $\times 84$ per cent 
$=9.2$ per cent of the prototrophs from the cross $\mathrm{Ch}^{\mathrm{r} 18} \times \mathrm{Ch}^{\mathrm{r} 22}$ should have a higher resistance than either parent, and should be mostly if not all of the Lac $+\mathrm{V}_{1}^{\mathrm{s}}$ phenotype. The actual results gave in prototrophs out of 205 (5.4 per cent) which were of resistance of $126 \mathrm{\gamma} / \mathrm{ml}$. and higher (that is practically double that of either resistant parent). This proportion of double-resistant recombinants (5.4 per cent) is in relatively good agreement with expectation ( 9.2 per cent) especially if one considers that the limits used for distinguishing between sensitives, resistants and double-resistants (less than 31 , between $3^{I}$ and 79 , more than $79 \gamma / \mathrm{ml}$.) are arbitrary and at least some resistants to $79 \mathrm{\gamma} / \mathrm{ml}$. of the $\mathrm{Chl}^{\mathrm{r} 18} \times \mathrm{Chl}^{\mathrm{r12}}$ cross might have been double-resistants, thus increasing the proportion of these by some units per cent. The expectation according to phenotype of double-resistants is at least partly confirmed, if one considers that of the 11 double-resistant prototrophs 6 show recombination, as expected, in the ist region and 5 in the other two regions on a total of 55 recombinations in the Ist region ( $\mathrm{M}-\mathrm{Lac}$ ) to 168 recombinations in the 2nd and 3 rd $\left(\mathrm{Lac}-\mathrm{V}_{1}\right.$ and $\left.\mathrm{V}_{1}-\mathrm{TL}\right)$.

\section{DISCUSSION}

It is possible to obtain higher and higher resistance to Chloromycetin in $E$. coli $\mathrm{K}-12$ by repeated transfers on growing concentrations of the drug, while a single exposure will result in the isolation of partially resistant individuals. The resistance acquired is relatively stable on subsequent cultivation in drug-free media but on prolonged culture it will often be lost unless special precautions are taken. However, loss of resistance requires months or years and the resistance levels are stable enough for short-term experiments.

This phenomenon of gradual adaptation, which is well known for many other drugs and micro-organisms, can be analysed genetically in the present case.

In principle the adaptation to Chl could be the result of one (or both) of the following mechanisms:

(i) physiological adaptation, by which resistance is developed gradually, the levels being comparable with the concentrations of drug employed during training. Hinshelwood (see for instance Dean and Hinshelwood) has proposed a model of gradual adaptation which could $a$ priori explain these results, provided one assumes that the changes in the enzyme-systems determined by contact with the drug, and which make the cells capable of withstanding the higher drug concentrations, are heritable in the absence of the drug for hundreds or thousands of generations;

(ii) a mutation and selection mechanism, in which case one can explain the existence of various steps of resistance by assuming

(a) a system of multiple alleles entailing various degrees of resistance;

(b) or preferably a polygenic system, which would more reason- 
ably account for the other fundamental aspect of this phenomenon, that in fact high resistance is never reached in a single step.

Mechanisms $(a)$ and $(b)$ can, however, co-exist.

Mechanism (b) was suggested by Demerec when he described the stepwise adaptation of Staphylococcus aureus to Penicillin.

Present experiments do not offer a direct answer to the question whether adaptation is of the physiological type (as assumed by Hinshelwood for other cases of drug resistance) or genetical, i.e. by mutation and selection. This question has been answered with more direct experiments (Cavalli, 1952) which were in favour of the second theory, thus confirming previous evidence for other drugs and organisms (Demerec, 1945; Oakberg and Luria, 1947; Newcombe and Hawirko, 1949; and others).

The present experiments however give a strong indirect support to the genetical theory, in that they show that wherever resistance is acquired, genetical analysis-by crossing to sensitives-shows segregations of gene-differences due to loci linked with the ordinary markers. Moreover, highly resistant strains show, on crossing to sensitives, all possible intermediate degrees of resistance, as expected if many genes are involved. When strains with intermediate resistance, obtained with one short exposure to the drug, are crossed to sensitives, the segregations obtained denounce only one or at most two gene-differences.

However, the point which is most strongly in favour of the polygenic theory is that such genes are usually linked with the ordinary chromosome markers.

A finer analysis of segregation from first steps shows that even when a small degree of resistance is involved, there is no inheritance of the blending type.

On crossing independent first steps together as well as crossing high resistants to sensitives, it is found that some 5 and probably more loci are involved.

As expected under the polygenic theory, resistance-genes which are not allelic (as shown by (i) the fact that when crossed together they give rise to a certain proportion of sensitives and also (ii) if crossed to marked sensitives they show different linkages with the markers) can on recombination add their effects, giving recombinants with a resistance clearly higher than that of either parent.

Therefore while these results are in good agreement with a polygenic theory they would not be easily explained on a physiological theory.

Some facts which may at first sight cause doubt on this interpretation will be discussed in detail:

(i) resistant strains are not very stable in the long run. This may seem like "deadaptation" in a system of physiological adaptation, but it must be observed that the loss of resistance occurs only after repeated passages and is relatively abrupt, which is rather in agreement with the 
hypothesis of loss of resistance by selective advantage of back mutants.

It must be stressed that a selective advantage of back mutants is to be expected, because resistant organisms grow poorly both on ordinary media and on media containing the drug: highly resistant organisms reach only I $/ 4$ to $\mathrm{I} / 2$ the saturation density which the normal parents will reach in the same conditions.

In order to keep resistant strains at the original level of resistance it is advisable to isolate at frequent intervals on drug-free media and test individual colonies for resistance, subculturing only from those which show the original level of resistance. If this is not done at frequent intervals it is likely that all the colonies tested will be of a lower level; on the other hand a selection on media containing the drug might introduce new unwanted genes for resistance.

(ii) In some of the crossing experiments no clear cut or no easily explainable linkage with the ordinary markers was found. This may mean that the loci involved are outside the marked region. A discussion of such results will however be premature, until the genetical system of $E$. coli $\mathrm{K}-12$ is more fully known.

(iii) There are several facts showing that selection favours positive interaction between genes, and recombination reveals negative interaction between them. Especially the first consideration is just to be expected. In fact selection builds a resistant polygenic system in this sequence:

$$
\mathrm{r}^{\circ} \text { (sensitive) }<\mathrm{r}^{1}<\mathrm{r}^{1} \mathrm{r}^{2}<\mathrm{r}^{1} \mathrm{r}^{2} \mathrm{r}^{3} \ldots
$$

where $<$ and $>$ stand for less or more resistant, and the following considerations are pertinent:

(a) this system is compatible with

$$
r^{2} r^{3}=r^{\circ} \text { (negative interaction between } r^{2}, r^{3} \text { if } r^{2}, r^{3}>r^{\circ} \text { ) }
$$

or even $\quad r^{2}=r^{\circ}, r^{3}=r^{\circ}\left(r^{2}, r^{3}\right.$ "modifiers" of $\left.r^{1}\right)$.

(b) of several second step mutants $r^{2}, r^{3}, r^{4}$ which may appear in a partially resistant clone $\mathrm{r}^{1}$, the double mutant favoured and therefore selected will be the one showing the highest positive interaction.

Therefore, when a polygenic system for resistance is broken down by recombination with a sensitive strain, resistance is expected to be low on average in the recombinants, and if two independently built polygenic systems are allowed to recombine together, positive interactions will disappear and negative interactions may be revealed with the result that resistance of recombinants will diminish on average.

If the results obtained by crosses of Chl resistant organisms could be generalised, one should think that the description of a polygenic system might require a number of parameters equal to the number of degrees of freedom involved; i.e. the number of possible genotypes minus one. This seems unfortunate but may be unavoidable. On the other hand, in the case of $\mathrm{Ch}^{\mathrm{r} 18}$ one locus seems to interact favourably 
with all other genes, with which it was brought together by recombination. If the interactional behaviours were at least for some genes a property of genes rather than of gene combinations, the number of parameters needed to describe a polygenic system might be reduced. As far as we know we can only say that such a number of parameters will be between a minimum, which is the number of loci $n$ involved in the polygenic system under consideration (and would be enough for a system where all interactions were additive on some suitable scale) and a maximum, which is $2^{n}$ - 1 for a haploid organism.

(iv) Selection for one character is known to be often accompanied by changes in other characters (Mather's "correlated response"). Although here no clear cut correlation between resistance and other morphological and biochemical characters has been found, it is worth mentioning some exceptions: in one case a first step resistant was found, giving highly disturbed segregations of the ordinary markers (an excess of $\mathrm{Lac}+\mathrm{V}_{1}^{\mathrm{r}}$ ). This may mean a chromosome mutation, or a mutation for auxotrophism accompanying Chl-resistance (not further tested). In other cases less striking disturbances of the segregations have been observed (see for instance a deficiency of $\mathrm{Lac}-\mathrm{V}_{1}^{\mathrm{s}}$ recombinants in table 2, cross I) and may have similar origins.

One typical morphological change observed in most if not all first or higher step resistants, is a mucoid appearance of the resistant colonies especially when grown on media rich in carbohydrates. There are various degrees of mucosity up to giant mucoid. Recombination between a mucoid resistant and a normal sensitive gives rise to segregation into four classes, of which mucoid sensitives and normal resistants are rarest. This is a genetical puzzle which has not been solved: no serious attempts were made, however, because of the highly variable expression of the mucoid character.

These results suggest that drug resistance in micro-organisms can offer an excellent field of study for quantitative inheritance. In fact, in bacteria, unlike higher organisms, single mutational steps can be isolated and analysed genetically. The possibility of analysis in biochemical terms of the effect of single genes is readier to hand, thus making an interpretation of interactions between different genes more easily attainable. However for such an analysis three conditions at least must be met: a fuller knowledge of the genetical system of bacteria, a drug with mechanism of action better known than that of Chl, and an accurate and simple method for measuring resistance.

\section{CONCLUSIONS AND SUMMARY}

I. Resistance to $\mathrm{Chl}$ (Chloromycetin) in $E$. coli $\mathrm{K}-\mathrm{I} 2$ is acquired only gradually. Short exposures secure only a moderate degree of resistance, repeated selection being necessary to reach high levels.

2. The genetical analysis has shown that a polygenic system is involved. In fact (i) on crossing high resistants to sensitives one finds recombinants showing all degrees of resistance between those of the 
parents; (ii) polygenes determining resistance are linked with the ordinary chromosome markers.

3. Recombinations between non-allelic independent first step resistants can result in addition of their resistances, so that prototrophs arise which are more resistant than either parent.

4. Repeated selection tends to build a polygenic system rich in positive interactions. Recombination is likely to break down such positively interacting systems and, by bringing together resistance genes which need not interact positively or even simply additively, may reveal important negative interactions.

5. These results are not easily compatible with a theory of physiological adaptation.

Acknowledgment.-We wish to take this opportunity to thank Professor R. A. Fisher for his interest and encouragement during this investigation.

\section{REFERENCES}

Cavalli, L. L. I950. La sessualitlà nei batteri. Boll. Ist. Sieroter. Mil., 29, 281-289. CAVAlli, L. L. 1952. Genetic analysis of drug resistance. Bull. World Hlth. Org., 6, I 85-206.

Cavalli, L. L., AND maccacaro, G. A. 1950. Chloromycetin-resistance in E. coli, a case of quantitative inheritance in Bacteria. Nature, I66, $991-992$.

DEAN, A. C. R., AND HINSHELWOOD, C. 195I. Induced and other variations in bacterial cultures. Part 1. The chemical basis of the changes. 7. Chem. Soc., I 157-I I59.

DEMEREc, M. 1945. Production of Staphylococcus strains resistant to various concentrations of penicillin. P.N.A.S., 3I, $16-24$.

DEMEREC, M. 1948. Origin of bacterial resistance to antibiotics. $\mathcal{7}$. Bact., $56,63-74$.

DEMEREc, M. 1949. Patterns of bacterial resistance to penicillin, aureomycin and streptomycin. J. Clin. Inv., 28, 891-893.

DEMEREC, M. 1950. Reaction of populations of unicellular organisms to extreme changes in environment. Amer. Nat., 84, 5-16.

EAST, E. M. 1915. Studies on size inheritance in Nicotiana. Genetics, 1, 164-176.

Fisher, R. A. 1918. The correlation between relatives on the supposition of Mendelian inheritance. Trans. R. Soc. Edinb., 52, 399-433.

LEA, D. E., AND COUlson, C. A. 1949. The distribution of the numbers of mutants in bacterial populations. 7. Genet., 49, 264-285.

LEDERBERG, J. 1947. Gene recombination and linked segregations in Escherichia coli. Genetics, 32, 505-525.

LEDERBERG, J. I950. The selection of genetic recombinations with bacterial growth inhibitors. $\mathcal{7}$. Bact., 59, $211-215$.

LEDERBERG, J., AND TATUM, E. L. 1946. Gene recombination in Escherichia coli. Nature, $158,55^{8}$.

Lerner, I. M. 1950. Population Genetics and animal improvement. Cambridge, I950.

LURIA, S. E., AND DELBRŬCK, M. 1943. Mutations of bacteria from virus sensitivity to virus resistance. Genetics, 28, 491-5I I.

MATHER, K. Biometrical Genetics. London.

MERKEL, J. R., HERMANN, E. G., AND STEERS, E. I951. A study of chloramphenicol resistance in Escherichia coli. Bact. Proc., 1951, 55.

NEWCOMBE, H. B., AND HAWIRKO, R. 1949. Spontaneous mutations to streptomycinresistance and dependence in Escherichia coli. $\mathcal{F}$. Bact., 57, 565-572.

NEWCOMBe, H. B., AND NYHolm, M. H. 1950a. The inheritance of streptomycin-resistance and dependence in crosses of Escherichia coli. Genetics, 35, 603-61 1. 
NEWCOMBE, H. B., AND NYHOLM, м. H. 1950 $b$. Anomalous segregations in crosses of Escherichia coli. Amer. Nat., 84, 457-465.

NILSSON-EHLE, H. 1909. Kreuzungsuntersuchungen an Hafer und Weizen. Lund.

OAKBERG, E. F., AND LURIA, S. E. 1947. Mutation to sulfonamide resistance in Staphilococcus aureus. Genetics, 32, 249-26r.

SMITH, G. N., AND WORREL, c. s. 1950. The decomposition of chloromycetin (chloramphenicol) by micro-organisms. Arch. Biochem., 28, 232-24I.

Note added in proof.-While this paper was in the press, a report of work by $\mathbf{R}$. D. Hotchkiss was published (Cold Spring Harbor Symposia, vol. xvI) showing that the transfer of heritable characters like various drug resistances can take place in Pneumococci by exposing in suitable conditions sensitive cells to cell-free, DNA-containing preparations obtained from resistant cells. While this has greatly amplified the meaning of the early transformation experiments in Pneumococci, it has made genetic analysis possible in essentially asexual organisms. In particular, the results given by Hotchkiss on the inheritance of penicillin resistance fit satisfactorily the scheme which we have also used to explain chloromycetin resistance. 